\section{COMPARISON OF TWO TECHNIQUES FOR ASSESSING PANCREATIC ISLET VIABILITY: FLOW CYTOMETRY AND FLUORESCEIN DIACETATE/PROPIDIUM IODIDE STAINING}

\author{
Natália Emerim Lemos ${ }^{1,2}$, Cristine Dieter ${ }^{1,2}$, Mariela Granero Farias ${ }^{3}$, \\ Jakeline Rheinheimer ${ }^{1,2}$, Bianca Marmontel de Souza ${ }^{1,2}$, Andrea Carla \\ Bauer $^{1,2}$, Daisy Crispim ${ }^{1,2}$
}

\begin{abstract}
Introduction: The success of islet transplantation for patients with unstable type 1 diabetes mellitus depends, in part, on the number of isolated islets and their quality, which is assessed by functional and viability tests. The test currently employed to evaluate islet viability, used by the Collaborative Islet Transplant Registry to release products for transplantation, is fluorescein diacetate/propidium iodide (FDA/PI) staining. However, the efficacy of this method relies on researcher experience; in this context, a quantitative method may be useful. The aim of this study was to compare islet viability as assessed by flow cytometry and the FDA/PI assay.
\end{abstract}

Methods: Viability was analyzed in islets isolated from 10 male Wistar rats. Upon FDA/PI staining, 50 islets from each animal were analyzed under fluorescence microscopy by two well-trained researchers. For flow cytometry, islets were dispersed and 100000 single cells were incubated with the 7-amino-actinomycin D (7AAD) fluorophore (dyes necrotic and late apoptotic cells) and the Annexin V-APC antibody (marks early apoptotic cells).

Results: A moderate correlation was found between techniques $(r=0.6 ; p=0.047)$. The mean islet viability measured by flow cytometry was higher than that estimated using FDA/PI staining (95.5 $\pm 1.4 \%$ vs $89.5 \pm 5.0 \% ; p=0.002)$.

Conclusions: Although flow cytometry is more expensive and time-consuming than FDA/PI staining, it is a quantitative technique with greater reproducibility that is less subject to inter-observer variability than FDA/PI. Therefore, flow cytometry appears to be the technique of choice when aiming for a more precise determination of islet viability.

Keywords: Islet transplantation; Islet isolation; Viability; Flow cytometry

\section{INTRODUCTION}

Pancreatic islet transplantation is an established treatment strategy for patients with type 1 diabetes mellitus that suffer from hypoglycemia unawareness and marked glycemic lability ${ }^{1-5}$. An important criterion for the achievement of long term insulin-independence with islet transplantation is the number of viable islets transplanted per kg of the recipient's weight ${ }^{6}$. It is generally assumed that a combined implant mass of at least 10,000 islet equivalents (IEQ) per kilogram $(\mathrm{kg}$ ) is required to routinely achieve insulin independence 1,7 .

At present, most islet isolation centers keep islets in culture for 24-48 hours prior to transplantation, allowing them to recover from the stress generated by the isolation process and also allowing the preparation of the recipient, which includes the administration of induction immunosuppressive therapy ${ }^{7-9}$. During this culture period, approximately $20 \%$ of total islet mass is lost, which may compromise the success of the transplant ${ }^{10}$. Studies have shown that
Clin Biomed Res. 2021;41(4):325-331

1 Laboratório de Biologia das llhotas Pancreáticas Humanas, Serviço de Endocrinologia, Hospital de Clínicas de Porto Alegre. Porto Alegre, RS, Brasil.

2 Programa de Pós-Graduação em Ciências Médicas: Endocrinologia, Faculdade de Medicina, Universidade Federal do Rio Grande do Sul. Porto Alegre, RS, Brasil.

3 Serviço de Diagnóstico Laboratorial, Unidade de Diagnóstico Especializado, Hospital de Clínicas de Porto Alegre. Porto Alegre, RS, Brasil.

Corresponding author: Daisy Crispim dcmoreira@hcpa.edu.br Serviço de Endocrinologia, Hospital de Clínicas de Porto Alegre Rua Ramiro Barcelos, 2350 90035-003, Porto Alegre, RS, Brasil. 
the loss of islets during the culture period is due, in part, to the apoptosis triggered along the process of procurement (due to the catecholamine storm during brain death and cold ischemia time) and the islet isolation process ${ }^{11-15}$.

Therefore, the assessment of islet viability is an important quality test for releasing isolated islets for transplantation. Currently, islet viability is assessed by transplant centers worldwide that participate in the Collaborative Islet Transplant Registry (CITR) using the standard deoxyribonucleic acid (DNA)-binding dye exclusion technique with fluorescein diacetate (FDA) and propidium iodide $(\mathrm{PI})^{7,16,17}$. In this method, living cells actively convert non-fluorescent FDA into the green fluorescent compound fluorescein, while dead cells show red fluorescence in their nuclei due to PI penetration through the permeabilized membrane ${ }^{18}$. A limitation of the FDA/PI staining method is that PI only enters cells that have lost selective membrane permeability, thus it does not dye early apoptotic cells ${ }^{17}$. Moreover, this method is not able to discriminate between cell subsets and, in particular, it does not allow selectively defining beta-cell viability 17,19 .

Therefore, alternative methods have been tested to determine islet viability before clinical transplantation, including flow cytometry ${ }^{17,19-23}$. By using different fluorescent dyes, flow cytometry may offer a more precise quantification of islet viability while also allowing the differentiation of cell death mechanisms (necrosis, early or late apoptosis). Another advantage of flow cytometry when compared to FDA/PI staining is that it is a quantitative method, not dependent on the operator to determine the percentage of living cells ${ }^{24,25}$. In this context, in the present study we compared islet viability percentages measured using flow cytometry and the FDA/PI assay.

\section{Methods}

\section{Animals and experimental design}

Ten male Wistar rats fed with a standard laboratory diet ad libitum and weighing 300 to $350 \mathrm{~g}$ were used in the study. All animals were kept in the animal facility of Hospital de Clínicas de Porto Alegre (Porto Alegre, Brazil) and were cared for according to guidelines for the use and care of laboratory animals ${ }^{26}$. The study was approved by the local Ethics Committee on Animal Use (Ethical approval number: 13-0166). A total of 10 consecutive islet preparations were analyzed for viability using the methods described below, with each animal corresponding to one islet preparation process. Approximately 500 islets were obtained in each preparation.

\section{Islet isolation and dispersion}

Islet isolation was performed following the protocol by Carter et al. ${ }^{27}$. After euthanasia, the bile duct was cannulated to allow pancreatic perfusion with $10 \mathrm{~mL}$ of cold Hank's balanced salt solution (HBSS, Sigma-Aldrich, Saint Louis, MO, USA) containing $0.5 \mathrm{mg} / \mathrm{mL}$ of collagenase P (Roche Diagnostics, Mannheim, Germany). The perfused pancreas was removed and digested for $15 \mathrm{~min}$ at $37^{\circ} \mathrm{C}$. Digestion was stopped by the addition of RPMI 1640 medium (11 mmol/L glucose) (Thermo Fisher Scientific, Gaithersburg, USA) supplemented with $10 \%$ fetal bovine serum (FBS), 100 units $/ \mathrm{mL}$ penicillin, $0.1 \mathrm{mg} /$ $\mathrm{mL}$ streptomycin, and $25 \mathrm{mmol} / \mathrm{L}$ HEPES (Thermo Fisher Scientific). Islet purification was performed with a discontinuous Histopaque density gradient (Sigma-Aldrich), at $1.119,1.100$, and $1.077 \mathrm{~g} / \mathrm{mL}$ density layers. An aliquot of purified islets was counted under a microscope to obtain the total number of isolated islets, which were then divided into two aliquots for further viability assessment by flow cytometry and FDA/PI staining.

\section{Islet viability assessment}

For FDA/PI staining, $43 \mu \mathrm{l}$ of precipitated islets isolated from each animal were stained with $0.46 \mu \mathrm{M}$ FDA and $14.34 \mu \mathrm{M} \mathrm{PI}$ (Sigma-Aldrich) in $457 \mu \mathrm{l}$ DPBS buffer (Sigma-Aldrich), being immediately analyzed by two well-trained researchers under a Nikon Eclipse TS100 microscope (Nikon, Tokyo, Japan) to estimate the percentage of living and dead cells in each islet. Each researcher analyzed 50 islets in suspension. Final islet viability was the mean percentage of living cells among the 50 islets analyzed by each researcher.

For islet viability assessment using flow cytometry, first we dispersed 100 islets into single cells. Isolated islets were immediately washed with HBSS (without calcium chloride and magnesium sulphate; Sigma-Aldrich) and dispersed with $0.125 \%$ trypsin-EDTA (Thermo Fisher Scientific) for 5 min at $37^{\circ} \mathrm{C}$. Dispersion was stopped by the addition of supplemented RPMI 1640 medium. Next, the newly obtained single cells were resuspended in phosphate buffered saline (PBS, LGC, Cotia, Brazil) $1 \times$ and Annexin V-APC binding buffer (BD, Franklin Lakes, USA). Then, $5 \mu \mathrm{L}$ of the Annexin V-APC antibody (labels early apoptotic cells; BD) was added to the cell preparation, which was incubated for $15 \mathrm{~min}$ at room temperature, protected from light. After incubation, $5 \mu \mathrm{L}$ of the 7AAD fluorophore (BD) was added to the cell suspension and 100,000 cells were acquired on a FacsCanto II flow cytometer (BD). Results were analyzed using Infinicyt software (Cytognos, Salamanca, Spain). Cells stained with both 7AAD and Annexin V-APC were those that had undergone membrane rupture through late apoptosis 
or necrosis. Cells marked only with Annexin V-APC had intact membranes but were already in the process of early apoptosis. Cells not stained with any dye were living cells.

\section{Statistical analysis}

Variables are shown as means \pm standard deviations (SDs) or percentages. Mean viability values obtained using flow cytometry or FDA/PI staining in the 10 islet isolation experiments were compared using Student's $t$-tests. The correlation between viability values obtained using both techniques was calculated using Pearson's correlation test. $p$ values $<0.05$ (two-tailed) were considered statistically significant, and all analyses were performed using SPSS 18 (SPSS Inc., Chicago, IL, USA).

$\mathbf{A}$
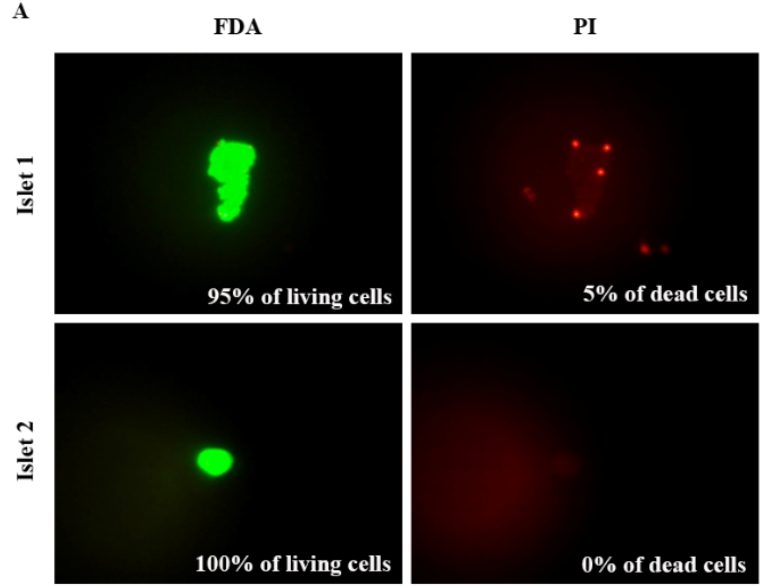

\section{RESULTS}

Figure $1 \mathrm{~A}$ illustrates the FDA/PI staining of two islets, where the green color (FDA staining) represents living cells inside an islet and the red color (PI staining) represents dead cells/islets. In this figure, islet 1 was considered as having $95 \%$ of living cells and $5 \%$ of dead cells, while islet 2 had $100 \%$ of living cells. Figure 1B shows the gating strategy used in flow cytometry for the assessment of islet viability after 7AAD and Annexin $\mathrm{V}$-APC treatment. The lower left quadrant shows living cells that did not stain with Annexin V-APC and $7 A A D$. The lower right quadrant shows cells undergoing early apoptosis (stained only with Annexin V-APC), while the upper right quadrant depicts cells suffering late apoptosis or necrosis (stained with both 7AAD and Annexin V-APC).

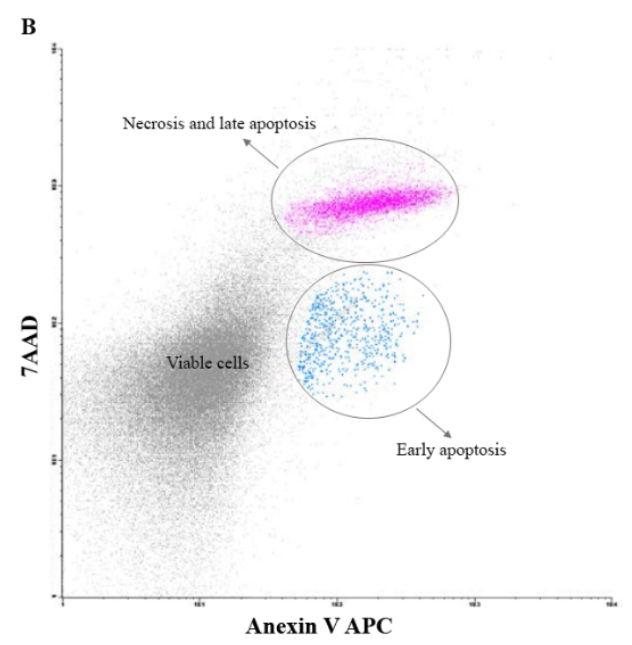

Figure 1: Representative images of fluorescein diacetate and propidium iodide (FDA/PI) and flow cytometry assays used for assessing islet viability. A: Rat pancreatic islet stained with FDA (green) and PI (red), showing the percentage of living (green) and dead (red) cells as estimated by a well-trained researcher; B: Gate strategies used to assess islet viability by flow cytometry using 7-amino-actinomycin D (7AAD) and Annexin V-APC. The islets were dissociated as single cells before treatment with 7AAD and Annexin V-APC. Approximately 100,000 cells were analyzed by this technique. Early apoptotic cells are marked only with Annexin V-APC, while necrotic/late apoptotic cells are marked with both 7AAD and Annexin V-APC.

Table 1 shows viability results (\%) obtained using flow cytometry and FDA/PI staining for each of the islet preparations isolated from the 10 Wistar rats. Regarding FDA/PI staining, 50 islets per isolation experiment were analyzed by each of the two researchers who estimated viability, and results are shown as mean percentages of living cells per islet isolation experiment (Table 1 and
Figure 1). Flow cytometry results are shown as mean percentages of viable cells, as well as the corresponding percentages of early apoptotic or late apoptotic/necrotic cells (Table 1), considering 100,000 single cells per experiment. The percentage of dead cells obtained by flow cytometry was the sum of the percentages of early apoptotic cells and late apoptotic/necrotic cells. 
Lemos et al.

Table 1: Viability assessed by fluorescein diacetate and propidium iodide (FDA/PI) staining and flow cytometry in each of the 10 analyzed experiments (EXP).

\begin{tabular}{cccccc}
\hline \multicolumn{7}{c}{ Type of death by flow cytometry } & & & \\
\hline EXP & Early apoptosis (\%) & $\begin{array}{c}\text { Necrosis + late } \\
\text { apoptosis (\%) }\end{array}$ & $\begin{array}{c}\text { Total death by flow } \\
\text { cytometry (\%) }\end{array}$ & $\begin{array}{c}\text { Viability by flow } \\
\text { cytometry (\%) }\end{array}$ & $\begin{array}{c}\text { Viability by } \\
\text { FDA/PI (\%) }\end{array}$ \\
\hline 1 & 0.56 & 5.20 & 5.76 & 94.24 & 88.50 \\
2 & 0.47 & 4.10 & 4.57 & 95.43 & 87.00 \\
3 & 1.38 & 0.61 & 1.99 & 98.01 & 96.00 \\
4 & 0.50 & 3.90 & 4.40 & 95.60 & 85.00 \\
5 & 1.17 & 4.50 & 5.67 & 94.33 & 91.40 \\
6 & 1.56 & 1.67 & 3.23 & 96.77 & 86.70 \\
7 & 0.90 & 2.96 & 3.86 & 96.14 & 97.10 \\
8 & 0.57 & 2.68 & 3.25 & 96.75 & 95.00 \\
9 & 1.10 & 5.50 & 6.60 & 93.40 & 84.40 \\
10 & 0.86 & 5.00 & 5.86 & 94.14 & 83.90 \\
\hline
\end{tabular}

For FDA/PI staining, islet viability in each experiment is the mean value estimated by two trained researchers.

Considering all experiments together, the mean islet viability obtained by FDA/PI staining was lower than that measured by flow cytometry $(89.5 \% \pm 5.03 \%$ vs $95.5 \% \pm 1.43 \%, p=0.002)$ (Figure 2). A moderate correlation was observed between mean viability percentages obtained by the techniques $(r=0.638, p=0.047)$. Of note, islet viability assessed by FDA/PI staining presented higher variability $(S D=5.03)$ than that assessed by flow cytometry $(S D=1.43)$, and this is mainly due to the variation in values obtained by each researcher: the SD between researchers ranged from 4.71 to $20.74 \%$.

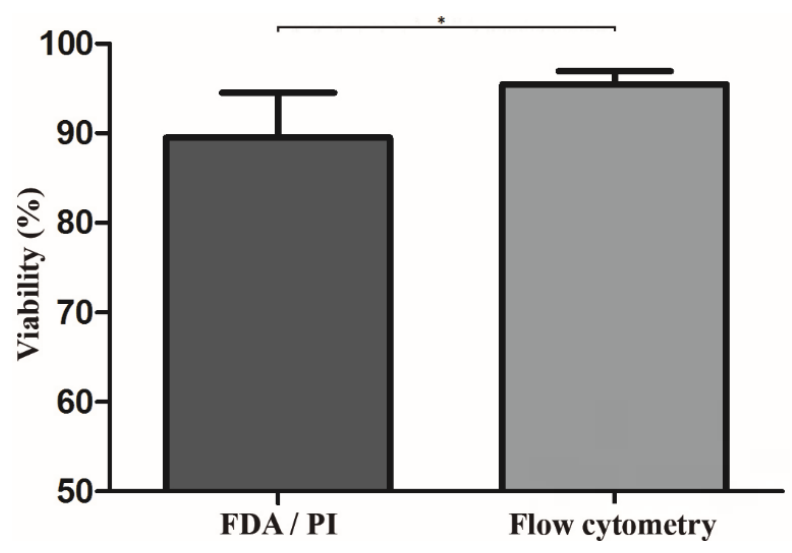

Figure 2: Mean viability percentages obtained using FDA/ $\mathrm{PI}$ staining and flow cytometry. Results are mean values for 10 experiments using each technique $\left({ }^{*} p=0.002\right)$.

\section{DISCUSSION}

Regulatory agencies require that the manufactured product of islet isolation be tested for sterility, purity, number of IEQs, function (glucosestimulated insulin secretion), and viability prior to release for transplantation ${ }^{7,9}$. An islet viability of over $70 \%$ is thus needed for the release of clinical islet products ${ }^{7}$. In centers belonging to the CITR, islet viability is commonly assessed by FDA/PI staining ${ }^{7,18}$, which is a simple and cheap technique but has some limitations. Therefore, in an effort to establish a more sensitive and specific method for evaluating islet viability, we compared viability measurements using FDA/PI staining or flow cytometry with 7AAD and Annexin V-APC. Our results demonstrated a significant difference between mean islet viability values assessed by these techniques, with flow cytometry showing less variation among experiments.

Although FDA/PI staining is the current standard method for islet viability estimation in the clinical transplantation setting, this assay has important limitations ${ }^{23}$. Firstly, the manual method for determining viability using FDA/PI is highly subjective, since usually two researchers estimate the percentages of living and dead cells under fluorescence microscopy, with the final result being the mean of their results ${ }^{19}$. Accordingly, in the present study, we reported that differences between FDA/ $\mathrm{PI}$-derived viability percentages obtained by each well-trained researcher ranged from 4.71 to $20.74 \%$, increasing SD. Even in centers that use softwarebased quantification of FDA/PI fluorescence during islet preparation, differences in exposure time and camera/software settings can also impact viability interpretations ${ }^{22}$. The greater sensitivity of image acquisition achieved with a CCD camera might lead to inadequate assessments merely due to the manipulation of exposure time or other image capturing parameters ${ }^{22}$.

Secondly, because FDA nonspecifically penetrates all living cells in the islet preparation, including islets and contaminating acinar cells, this complicates the accurate estimation of islet viability ${ }^{19}$. Thirdly, the non-fluorescent FDA is converted into green 
fluorescent fluorescein dye by an esterase in the cytoplasm of living cells. However, some dead cells may still exhibit residual esterase activity, interfering with the identification of dead cells that show staining with both dyes ${ }^{22}$. In addition, PI only enters cells that have lost selective membrane permeability and is not able to identify early apoptotic cells, which still display intact membrane permeability ${ }^{17,19}$. Fourthly, several studies have shown that the FDA/PI viability assay does not correlate well with post-transplantation islet function ${ }^{19,22,28,29}$. For example, Papas et al. ${ }^{29}$ demonstrated that it was not suitable for predicting diabetes reversal in nude mice transplanted with human islets when compared to the measurement of fractional viability using the oxygen consumption rate normalized to DNA (OCR)/DNA. Islets with high OCR/DNA had higher chances of reversing diabetes in mice than islets with low OCR/DNA, despite the fact that both

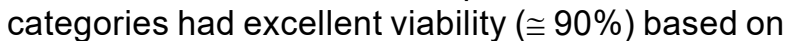
the FDA/PI assay. Finally, other issues related to the stability of dyes in storage, stock solutions, and incubation times may also influence the intensity of cell staining, thus influencing the final scoring of viability ${ }^{22}$. These limitations suggest that the FDA/PI assay may not be the optimal approach to assess islet viability, and alternative methods should be tested.

In comparison with the FDA/PI assay, flow cytometry is an automated technique that provides more accurate measurements with specific antibodies that can detect rare populations in a cell group ${ }^{24,25}$. When it comes to islet preparations, flow cytometry allows the identification of cells suffering early apoptosis, which are counted as living cells when assessed by FDA/PI staining. The correct identification of living cells is a topic of great importance since the number of functional transplanted islets is highly correlated with transplant success ${ }^{17,23,30}$. Even though flow cytometry is more precise than FDA/ $\mathrm{PI}$ staining in estimating islet viability, it is a more expensive technique considering the required dyes/ antibodies and cytometry equipment. Moreover, it is more time-consuming ( $\cong 2 \mathrm{~h}$ compared to $\cong 30-45$ min for FDA/PI), but it could be done timely during the final evaluation of the islet product before the release for islet transplantation. In addition, some studies ${ }^{17,21}$ use flow cytometry to assess the viability of each islet cell type with other markers, such as Newport Green PDX acetoxymethyl ether (NG) and tetramethylrhodamine ethyl ester (TMRE). However, the detection of variability according to different cell types or specifically considering betacells requires more complex and time-consuming techniques, as well a separator cytometer. Therefore, we opted for a technique that was more similar to FDA/PI staining, although more accurate. Based on the current viability threshold used for clinical islet product release ( $\geq 70 \%$ of living islets), both tested techniques would have obtained approval for islet product transplantation. However, evidence accumulated in the last few years has shown that even small increments in islet viability measured with techniques other than FDA/PI are associated with better islet transplantation outcomes ${ }^{22,28,31-33}$.

In conclusion, although flow cytometry is a more expensive and time-consuming technique than FDA/PI assay, it is more accurate and informative since the use of 7AAD and Annexin V-APC allows the differentiation of living islets, early apoptotic islets, and islets that already underwent apoptosis/ necrosis. The differentiation among these cells may be useful to better understand the quality of islet recovery after culture. Therefore, we believe that islet viability assessment using flow cytometry should be the technique of choice to when releasing islets for transplantation.

\section{Conflicts of interest}

The authors declare no conflicts of interest.

\section{Ethical approval}

This study was approved by the Ethics Committee on Animal Use (CEUA) from Hospital de Clínicas de Porto Alegre (Ethical approval number: 13-0166), Rio Grande do Sul, Brazil.

\section{Acknowledgments}

This study was partially supported by grants from Conselho Nacional de Desenvolvimento Científico e Tecnológico (CNPq), Fundo de Incentivo à Pesquisa e Eventos (FIPE) of Hospital de Clínicas de Porto Alegre, Fundação de Amparo à Pesquisa do Estado do Rio Grande do Sul (FAPERGS), and Coordenação de Aperfeiçoamento de Pessoal de Nível Superior (CAPES). D.C. is recipient of a CNPq scholarship. 


\section{REFERENCES}

1. Barton FB, Rickels MR, Alejandro R, Hering BJ, Wease S, Naziruddin B, et al. Improvement in outcomes of clinical islet transplantation: 1999-2010. Diabetes Care. 2012;35(7):1436-45.

2. Shapiro AM, Pokrywczynska M, Ricordi C. Clinical pancreatic islet transplantation. Nat Rev Endocrinol. 2017;13(5):268-77.

3. Brennan DC, Kopetskie HA, Sayre PH, Alejandro R, Cagliero E, Shapiro AM, et al. Long-Term FollowUp of the Edmonton Protocol of Islet Transplantation in the United States. Am J Transplant. 2016;16(2):509-17.

4. Lablanche S, Borot S, Wojtusciszyn A, Bayle F, Tétaz R, Badet L, et al. Five-Year Metabolic, Functional, and Safety Results of Patients With Type 1 Diabetes Transplanted With Allogenic Islets Within the Swiss-French GRAGIL Network. Diabetes Care. 2015;38(9):1714-22.

5. Hering BJ, Clarke WR, Bridges ND, Eggerman TL, Alejandro R, Bellin MD, et al. Phase 3 Trial of Transplantation of Human Islets in Type 1 Diabetes Complicated by Severe Hypoglycemia. Diabetes Care. 2016;39(7):1230-40.

6. Mineo D, Pileggi A, Alejandro R, Ricordi C. Point: steady progress and current challenges in clinical islet transplantation. Diabetes Care. 2009;32(8):1563-9.

7. Ricordi C, Goldstein JS, Balamurugan AN, Szot GL, Kin T, Liu C, et al. National Institutes of HealthSponsored Clinical Islet Transplantation Consortium Phase 3 Trial: Manufacture of a Complex Cellular Product at Eight Processing Facilities. Diabetes. 2016;65(11):3418-28.

8. Kempf MC, Andres A, Morel P, Benhamou PY, Bayle F, Kessler L, et al. Logistics and transplant coordination activity in the GRAGIL Swiss-French multicenter network of islet transplantation. Transplantation. 2005;79(9):1200-5.

9. Rheinheimer J, Bauer AC, Silveiro SP, Estivalet AA, Bouças AP, Rosa AR, et al. Human pancreatic islet transplantation: an update and description of the establishment of a pancreatic islet isolation laboratory. Arch Endocrinol Metab. 2015;59(2):161-70.

10. Kin T, Senior P, O'Gorman D, Richer B, Salam A, Shapiro AM. Risk factors for islet loss during culture prior to transplantation. Transpl Int. 2008;21(11):1029-35.

11. Contreras JL, Eckstein C, Smyth CA, Sellers MT, Vilatoba M, Bilbao G, et al. Brain death significantly reduces isolated pancreatic islet yields and functionality in vitro and in vivo after transplantation in rats. Diabetes. 2003;52(12):2935-42.

12. Korsgren $\mathrm{O}$, Lundgren $\mathrm{T}$, Felldin $\mathrm{M}$, Foss A, Isaksson B, Permert J, et al. Optimising islet engraftment is critical for successful clinical islet transplantation. Diabetologia. 2008;51(2):227-32.

13. Biarnes M, Montolio M, Nacher V, Raurell M, Soler J, Montanya E. Betacell death and mass in syngeneically transplanted islets exposed to short- and long-term hyperglycemia. Diabetes. 2002;51(1):66-72.

14. Cattan P, Berney T, Schena S, Molano RD, Pileggi A, Vizzardelli $C$, et al. Early assessment of apoptosis in isolated islets of Langerhans. Transplantation. 2001;71(7):857-62.

15. Abdelli S, Ansite J, Roduit R, Borsello T, Matsumoto I, Sawada T, et al. Intracellular stress signaling pathways activated during human islet preparation and following acute cytokine exposure. Diabetes. 2004;53(11):2815-23.

16. London NJ, Contractor H, Lake SP, Aucott GC, Bell PR, James RF. A microfluorometric viability assay for isolated human and rat islets of Langerhans. Diabetes Res. 1989;12(3):141-9.

17. Ichii $H$, Inverardi L, Pileggi $A$, Molano RD, Cabrera O, Caicedo A, et al. A novel method for the assessment of cellular composition and beta-cell viability in human islet preparations. $A m$ J Transplant. 2005;5(7):1635-45.

18. NIH CIT Consortium Chemistry Manufacturing Controls Monitoring Committee; NIH CIT Consortium. Purified Human Pancreatic
Islet - Viability Estimation of Islet Using Fluorescent Dyes (FDA/PI): Standard Operating Procedure of the NIH Clinical Islet Transplantation Consortium. CellR4 Repair Replace Regen Reprogram. 2015;3(1):e1378.

19. Komatsu H, Omori K, Parimi M, Rawson J, Kandeel F, Mullen Y. Determination of Islet Viability Using a Zinc-Specific Fluorescent Dye and a Semiautomated Assessment Method. Cell Transplant. 2016;25(10):1777-86.

20. Jayaraman S. A novel method for the detection of viable human pancreatic beta cells by flow cytometry using fluorophores that selectively detect labile zinc, mitochondrial membrane potential and protein thiols. Cytometry A. 2008;73(7):615-25.

21. Iglesias I, Bentsi-Barnes K, Umeadi C, Brown L, Kandeel F, Al-Abdullah IH. Comprehensive analysis of human pancreatic islets using flow and laser scanning cytometry. Transplant Proc. 2008;40(2):351-4.

22. Boyd V, Cholewa OM, Papas KK. Limitations in the Use of Fluorescein Diacetate/Propidium lodide (FDA/ $\mathrm{PI}$ ) and Cell Permeable Nucleic Acid Stains for Viability Measurements of Isolated Islets of Langerhans. Curr Trends Biotechnol Pharm. 2008;2(2):66-84

23. Barnett MJ, McGhee-Wilson D, Shapiro AM, Lakey JR. Variation in human islet viability based on different membrane integrity stains. Cell Transplant. 2004;13(5):481-8.

24. Steen HB, Lindmo T. Flow cytometry: a high-resolution instrument for everyone. Science. 1979;204(4391):403-4.

25. Darzynkiewicz Z, Li X, Bedner E. Use of flow and laser-scanning cytometry in analysis of cell death. Methods Cell Biol. 2001;66:69-109.

26. National Research Council (US), Committee for the Update of the Guide for the Care and Use of Laboratory Animals. Guide for the Care and Use of Laboratory Animals. 8th ed. Washington (DC): National Academies Press;2011.

27. Carter JD, Dula SB, Corbin KL, Wu R, Nunemaker CS. A practical guide to rodent islet isolation and assessment. Biol Proced Online. 2009;11:3-31. 
28. Ricordi C, Lakey JR, Hering BJ. Challenges toward standardization of islet isolation technology. Transplant Proc. 2001;33(1-2):1709.

29. Papas KK, Colton CK, Nelson RA, Rozak PR, Avgoustiniatos ES, Scott WE 3rd, et al. Human islet oxygen consumption rate and DNA measurements predict diabetes reversal in nude mice. Am J Transplant. 2007;7(3):707-13.
30. Hanson MS, Park EE, Sears ML, Greenwood KK, Danobeitia JS, Hullett DA, et al. A simplified approach to human islet quality assessment. Transplantation. 2010;89(10):1178-88.

31. Papas KK, Bellin MD, Sutherland DE, Suszynski TM, Kitzmann JP, Avgoustiniatos ES, et al. Islet Oxygen Consumption Rate (OCR) Dose Predicts Insulin Independence in Clinical Islet Autotransplantation. PLoS One. 2015;10(8):e0134428.
32. Kitzmann JP, O'Gorman D, Kin T, Gruessner AC, Senior P, Imes S, et al. Islet oxygen consumption rate dose predicts insulin independence for first clinical islet allotransplants. Transplant Proc. 2014;46(6):1985-8.

33. Eckhard M, Brandhorst D, Winter D, Jaeger C, Jahr H, Bretzel RG, et al. The role of current product release criteria for identification of human islet preparations suitable for clinical transplantation. Transplant Proc. 2004;36(5):1528-31.

Received: Oct 5, 2020 Accepted: Aug 2, 2021 